\title{
Limited diagnostic facilities impending the therapeutic approach of Mucopolysaccharidosis in Bangladesh: A case report
}

\author{
Orindom Shing Pulock ${ }^{1}$, Susmita Dey Pinky ${ }^{1}$, and Syeda Humaida Hasan ${ }^{1}$ \\ ${ }^{1}$ Chattogram Medical College and Hospital
}

February 3, 2022

\begin{abstract}
In resource-constrained settings, mucopolysaccharidosis (MPS) is a rare hereditary metabolic illness that frequently remains undiagnosed. We present a scenario that illustrates the challenges in diagnosing and managing MPS due to test inaccessibility, as well as potential approaches to minimize the hurdles.
\end{abstract}

\section{Introduction:}

The mucopolysaccharidoses (MPS) are a set of rare genetic disorders characterized by a lack of or malfunctioning of enzymes that metabolize glycosaminoglycans (GAGs) at the lysosomal level. They account for fewer than 0.1 percent of all genetic disorders. ${ }^{1,2}$ MPS can be found in various forms worldwide. Coarse facial characteristics, cognitive retardation, hepatosplenomegaly, hernias, kyphoscoliosis, corneal clouding, and other clinical symptoms vary depending on the individual enzyme deficit. Different MPS exhibit comparable symptoms, especially MPS I and II; however, there are some differences, such as severe neurological issues in MPS III and hydrops fetalis in MPS VII. ${ }^{3}$ These clinical signs help diagnose, but early and precise diagnosis in the asymptomatic stage is critical for better outcomes. The diagnosis is confirmed by identifying the malfunctioning enzyme and searching for a mutation. For patient management and family screening, it is essential to recognize mucopolysaccharidosis. Novel approaches such as urinary and blood GAG tests, enzyme assays, and gene tests aid in diagnosing and subtyping MPS. ${ }^{3,4}$ Based on experimental and clinical investigations, hematopoietic stem cell transplantation and enzyme replacement treatment have been established in the previous three decades. ${ }^{5}$

MPS is divided into seven types, and eleven subtypes differentiated biochemically by the related enzyme deficit. The incidence of MPS varies depending on the geographic location. For instance, MPS II is the most prevalent in the study site (Asia), whereas in Europe, MPS I \& MPS III are more prevalent. ${ }^{3}$ MPS II or Hunter syndrome is the only X-linked-inherited mucopolysaccharidosis primarily affecting males with an incidence rate ranging from 0.38 to 1.09 per 100,000 live male births. However, no documented study in Bangladesh shows the prevalence of Hunter's disease. ${ }^{6}$ Besides, both MPS I and II present with hepatosplenomegaly, cognitive retardation, Dystosis multiplex (large dolichocephalic skull, thickened calvarium with hyperostosis of the cranium, boot-shaped Sella tursica, thickened clavicles, ovoid vertebral bodies with a peak like projection, tapering of the terminal phalanges, flared ribs, etc.), dysmorphic appearance, corneal clouding, and cardiac abnormalities. Additionally, MPS II has features of dermatological signs. MPS III has limited CNS involvement features and probable Dystosis multiplex and dysmorphic appearances ${ }^{7,8}$.

Confirmatory diagnosis of MPS can be made by urinary and blood GAGs test, enzyme assay, and gene test; moreover, this can be diagnosed antenatally by fibroblast enzymatic activity in amniotic fluid and marke d GAGs storage in fetal organs ${ }^{9,10}$. A newborn screening program by enzymatic assay is recommended in the USA, Taiwan, and Italy. In the rest of the world, the newborn screening program doesn't exist. The 
physicians are confronted with the issue of identifying MPS patients in the early stages of the disease before irreversible damage has occurred. ${ }^{11}$ Moreover, most of these enzymatic studies are unavailable in resourcelimited centers such us in Bangladesh. Hence a careful and critical approach with supportive imaging features of the skeletal survey following patient's clinical features is essential for diagnosing MPS types ${ }^{12,13}$. Attempts had been to diagnose the MPS types without the biochemical test. A mnemonic screening tool for MPS II (Hernia/Hearing, Unusual faces, Nasal obstruction, Tongue and Tonsils, enlarged liver and spleen, Respiration/Range of Motion) (Mnemonic - HUNTER) was developed by the Hunter Outcome Survey (HOS) group. This tool is $95 \%$ accurate for diagnosing MPS II or Hunter syndrome. ${ }^{14}$ Additionally, few researchers from a neighboring country (Nepal) diagnosed MPS II from history, clinical features, and skeletal survey as they lack biochemical testing facilities in their limited-resource settings. ${ }^{12,15}$

Managing MPS entails halting the disease's severity and enhancing one's quality of life. Patients with MPS have several therapy choices, including palliative care, surgery, and disease-specific treatments such as Enzyme Replacement Therapy (ERT) and Hematopoietic Stem Cell Transplantation (HSCT). ${ }^{3}$ However, to accept the treatment, detailed clinical evaluation and disease history must be considered ${ }^{5}$. Although novel treatments such as substrate reduction therapy, pharmacological chaperone therapy, and gene therapy are becoming clinically available, early diagnosis is crucial to avail these treatment facilities. Many of these therapies, especially enzyme replacement therapy, have demonstrated the potential of alleviating the outcome and significantly raising the quality of life if started early in the disease ${ }^{5}$. Overall, clinical evaluation, early identification, and rapid diagnosis of the cases are the key to a better outcome since the treatment in these cases is time-sensitive.

\section{Case presentation:}

An 11-year-old South Asian boy was born to healthy, non-consanguineous parents as their middle child who presented to the pediatric in-patient department with lethargy, poor feeding of two months duration, and respiratory distress of two days. On inquiry, the patient had a history of difficulty swallowing both liquid and solid foods developed over two months, leading to severe dehydration. According to the parent's statement, they noticed a delay in his developmental milestones for the first time when he could not sit properly even at the age of 9 months. Afterward, a history of repeated respiratory infections was mentioned, although he never required any admission into hospitals for this. The parents also complained of the child's disturbed sleep pattern and agitated behavior. He was unable to communicate verbally, and so never attended any school. However, the patients' attendants mentioned that before this episode of his hospitalization, he used to have a good appetite and could sit and walk some distance independently.

Nevertheless, he did not develop any bowel or bladder control to date. There was no significant family history, and his siblings were affected. The patient had no history of convulsion, loss of consciousness, bleeding manifestation, jaundice, vomiting, constipation, or diarrhea.

There was a manifestation of growth retardation and coarse facial features [Figure 1(a)] . The patient had an occipitofrontal circumference (OFC) of 58 centimeters, frontal bossing, thick eyebrows, depressed nasal bridge, broad lip, malocclusion of teeth[Figure 1(a)], oral ulcer, short neck, and tiny stubby fingers [Figure $\mathbf{1}(\mathbf{c}, \mathbf{d})$ ] . No clouding of the cornea was observed. Expert opinion from the ophthalmology department was taken, and no abnormality was detected in the cornea, fundus, or lens.

The patient had hepatomegaly, $6 \mathrm{~cm}$ measuring from the subcostal margin in the right midclavicular line with a palpable spleen and an umbilical hernia [Figure 1(b)] . Palpatory and auscultatory findings of bronchopneumonia were also present.

A chest X-ray revealed spatulated ribs (tapering of the posterior end of the rib) regarding the imaging factors. The right dome of the diaphragm was $4 \mathrm{~cm}$ higher than the left [Figure 2(a)] . Hand wrist radiograph showed proximal tapering of metacarpal bones (bullet shape) and fusion of interphalangeal joints [Figure 2(b)]. The clinical aspects of this presenting case are summarized in Table 1.

Investigations after his admission to the hospital manifested severe hypernatremia $(168 \mathrm{mEq} / \mathrm{L})$ with hyper- 
chloremia (132 mEq/L). Complete blood count, Thyroid-stimulating hormone, and Ft4 were within normal range. Ultrasonography of the whole abdomen reported no abnormality, and kidney and liver function were normal. Due to the financial constraints and lack of testing facility, no urinalysis and echocardiography were done. The clinical evaluation and radiological findings made a probable diagnosis of Mucopolysaccharidosis II (Hunter Syndrome).

\section{Discussion:}

This case report describes a case of an 11 years old boy diagnosed with probable MPS II from the clinical correlations and radiological findings in a resource-limited setting where the confirmatory biochemical tests were not available. Since Infants with attenuated MPS appear pretty normal at birth, and early developmental milestones may also be typical. At the same time, some signs and symptoms overlap with common childhood complaints; often, MPS can stay undiagnosed until a later part of their childhood. ${ }^{16}$ In our case, the patient showed a delay in reaching his early developmental milestones and developed characteristic coarse facial features with repeated visits at the primary care center with recurrent respiratory tract infection. The possibility of a rare and genetic disease could not be investigated even after the presence of multiple red flag signs ${ }^{16}$ that a single illness could explain otherwise.

The presentation of this case is consistent with the expression of MPS I \& II in Table 1. However, he did not have any corneal change consistent with his MPS II diagnosis ${ }^{16}$. Previous cases reported with tonsillitis, adenoids, polyp presence on the laryngeal inlet, polypoid lesions, supraglottic narrowing, thickening of tongue, oropharyngeal mucosa due to deposition of glycosaminoglycans (GAG) explained the rationality of the dysphagia and sleep apnea in our patient. ${ }^{17}$ Structural involvement of his heart and brain couldn't be detected as no echocardiography, computed tomography, and magnetic resonance imaging tests were done. A screening test to diagnose mucopolysaccharidosis biochemically (urine for GAG test) and enzyme assay was initially planned to do. Still, it couldn't be implemented due to the unavailability and financial constraint of the family. After nine days of his hospital stay, the family was discharged against medical advice.

Even though this patient did not have the financial capability to afford the definite treatment of the disease and thus only received supportive care, still the rationality of treating this patient with ERT or HSCT remained open to debate since the efficacy of ERT and HSCT is presumably better in the early inception of the disease. ${ }^{18}$ However, studies denoted that MPS II patients, irrespective of the age of presentation and CNS involvement, should receive ERT that may attenuate the disease's severity. ${ }^{19}$

This case depicts a dilemma for the patient, his family members and the physicians. His family and the physician have faced that lack of testing facility led to delayed diagnosis at an older age, which hampers the capability of available treatment to work as the irreversible changes have already occurred. Therefore, improve disease recognition and early diagnosis through precise clinical evaluation, especially in resourcelimited settings is at utmost necessity. The stakeholders at resource-limited stages can follow the guideline to categorize possible MPS patients into high-risk and low-risk groups so that screening tests at an early age can be implemented ${ }^{11}$. In turn, this will help patients avail the novel therapeutics, hematopoietic stem cell transplantation, or enzyme replacement therapy that are likely to increase survival rates while significantly improving their quality of life. Moreover, initiatives should be taken to invest more in research to discover novel, cost-effective, and sustainable treatment approaches, which may act even in elder age.

\section{List of abbreviations}

MPS : Mucopolysaccharidosis

GAGs : Glycosaminoglycans

IDS : Iduronate-2-sulfatase

ERT : Enzyme replacement therapy

HSCT : Hematopoietic stem cell transplantation 
CNS : Central nervous system

OFC : Occipito-frontal Circumference

\section{Availability of data and materials}

The data used to support the findings of this study are included within the article.

\section{Conflict of interest}

Submitting authors are responsible for coauthors declaring their interests.

\section{Funding}

The authors declare that they have received no funding.

\section{Acknowledgments}

We thank our patient's parents for their cooperation and for providing consent for the publication and appreciate the continuous support from the Department of Pediatrics, Chattogram Medical College Hospital.

\section{Author Contributions:}

Orindom Shing Pulock: collected data, contributed to analysis and interpretation of data, and drafted the article and was involved in the care of the patient.

Susmita Dey Pinky: collected data, contributed to analysis and interpretation of data, and drafted the article and was involved in the care of the patient.

Syeda Humaida Hasan: contributed to analysis and interpretation, and drafted and critically revised the article.

\section{References}

1. Suarez-Guerrero JL, Gómez Higuera PJI, Arias Flórez JS, Contreras-García GA. [Mucopolysaccharidosis: clinical features, diagnosis and management]. Rev Chil Pediatr . 2016;87(4):295-304. doi:10.1016/j.rchipe.2015.10.004

2. Gaffke L, Pierzynowska K, Podlacha M, Brokowska J, Wegrzyn G. Changes in cellular processes occurring in mucopolysaccharidoses as underestimated pathomechanisms of these diseases. Cell Biol Int . 2021;45(3):498-506. doi:10.1002/cbin.11275

3. Zhou J, Lin J, Leung WT, Wang L. A basic understanding of mucopolysaccharidosis: Incidence, clinical features, diagnosis, and management. Intractable Rare Dis Res . 2020;9(1):1-9. doi:10.5582/irdr.2020.01011

4. Michaud M, Belmatoug N, Catros F, et al. Mucopolysaccharidoses : quand y penser ? La Revue de Médecine Interne . 2020;41(3):180-188. doi:10.1016/j.revmed.2019.11.010

5. Stapleton M, Hoshina H, Sawamoto K, et al. Critical review of current MPS guidelines and management. Mol Genet Metab . 2019;126(3):238-245. doi:10.1016/j.ymgme.2018.07.001

6. D'Avanzo F, Rigon L, Zanetti A, Tomanin R. Mucopolysaccharidosis Type II: One Hundred Years of Research, Diagnosis, and Treatment.International Journal of Molecular Sciences . 2020;21(4):1258. doi:10.3390/ijms21041258

7. Wraith JEd. Chapter 102 - Mucopolysaccharidoses. In: Rimoin D, Pyeritz R, Korf B, eds. Emery and Rimoin's Principles and Practice of Medical Genetics . Academic Press; 2013:1-40. doi:10.1016/B978-0-12383834-6.00107-5

8. Matalon R, Matalon KM. Mucopolysaccharidoses. In: Aminoff MJ, Daroff RB, eds. Encyclopedia of the Neurological Sciences . Academic Press; 2003:237-241. doi:10.1016/B0-12-226870-9/01991-2 
9. Torres R de O, Pintor AVB, Guedes FR, et al. Oral and craniofacial manifestations in a Hunter syndrome patient with hematopoietic stem cell transplantation: A case report. Spec Care Dentist . 2018;38(1):51-54. doi: $10.1111 /$ scd. 12260

10. Chen CP, Lin SP, Tzen CY, et al. Prenatal diagnosis and genetic counseling of mucopolysaccharidosis type II (Hunter syndrome). Genet Couns . 2007;18(1):49-56.

11. Kubaski F, de Oliveira Poswar F, Michelin-Tirelli K, et al. Diagnosis of Mucopolysaccharidoses. Diagnostics . 2020;10(3):172. doi:10.3390/diagnostics10030172

12. Shah GS, Mahal T, Sharma S. Atypical clinical presentation of mucopolysaccharidosis type II (Hunter syndrome): a case report. J Med Case Rep . 2010;4:154. doi:10.1186/1752-1947-4-154

13. Rasheeedah I, Patrick O, Abdullateef A, Mohammed A, Sherifat K, Gbadebo I. Challenges in the Management of Mucopolysaccharidosis Type II (Hunter's Syndrome) in a Developing Country: a Case Report. Ethiop J Health Sci . 2015;25(3):279-282. doi:10.4314/ejhs.v25i3.12

14. Cohn GM, Morin I, Whiteman DAH, on behalf of the Hunter Outcome Survey Investigators. Development of a mnemonic screening tool for identifying subjects with Hunter syndrome. Eur J Pediatr . 2013;172(7):965970. doi:10.1007/s00431-013-1967-x

15. Rayamajhi A, Pokharel PJ, Chapagain R, Rayamajhi AK. Mucopolysaccharidosis type II with inguinal hernia. J Nepal Health Res Counc . 2013;11(25):293-295.

16. Burton BK, Giugliani R. Diagnosing Hunter syndrome in pediatric practice: practical considerations and common pitfalls. Eur J Pediatr . 2012;171(4):631-639. doi:10.1007/s00431-012-1703-y

17. Suzuki K, Sakai H, Takahashi K. Perioperative airway management for aortic valve replacement in an adult with mucopolysaccharidosis type II (Hunter syndrome). JA Clin Rep . 2018;4(1):24. doi:10.1186/s40981-018-0162-5

18. Orchel-Szastak K, Ptak K, Hrnciar K, Pilch B, Kania U, Bik-Multanowski M. [Early enzyme replacement therapy - hope for patients with mucopolysaccharidosis Type II]. Pediatr Endocrinol Diabetes Metab . 2017;23(2):111-116. doi:10.18544/PEDM-23.02.0082

19. Christianto A, Watanabe H, Nakajima T, Inazu T. Idursulfase enzyme replacement therapy in an adult patient with severe Hunter syndrome having a novel mutation of iduronate-2-sulfatase gene. Clin Chim Acta . 2013;423:66-68. doi:10.1016/j.cca.2013.04.022

Table 1: Presenting features of the case in compare to the features of MPS I, II and III.

\section{Signs}

Coarse Facial features

Macrocephaly

Communicating hydrocephalus

Dental abnormalities

Cognitive/ developmental delay

Spinal cord compression

Carpal tunnel syndrome

Hyperactivity, aggression, impulsivity

Seizures

Hearing loss

Recurrent ear infection

Persistent rhinorrhea

Frequent respiratory infections

Respiratory obstruction 
Sleep apnea

Hepatosplenomegaly

Umbilical hernia

Chronic diarrhea

Dystosis multiplex

Growth retardation

Claw hands

Joint stiffness

Cardiac valve disease

Corneal clouding

"+" = Present, "-" = Absent

Structure of this table is modified upon the work of Burton, B.K., Giugliani, R. Diagnosing Hunter syndrome in pediatric p 

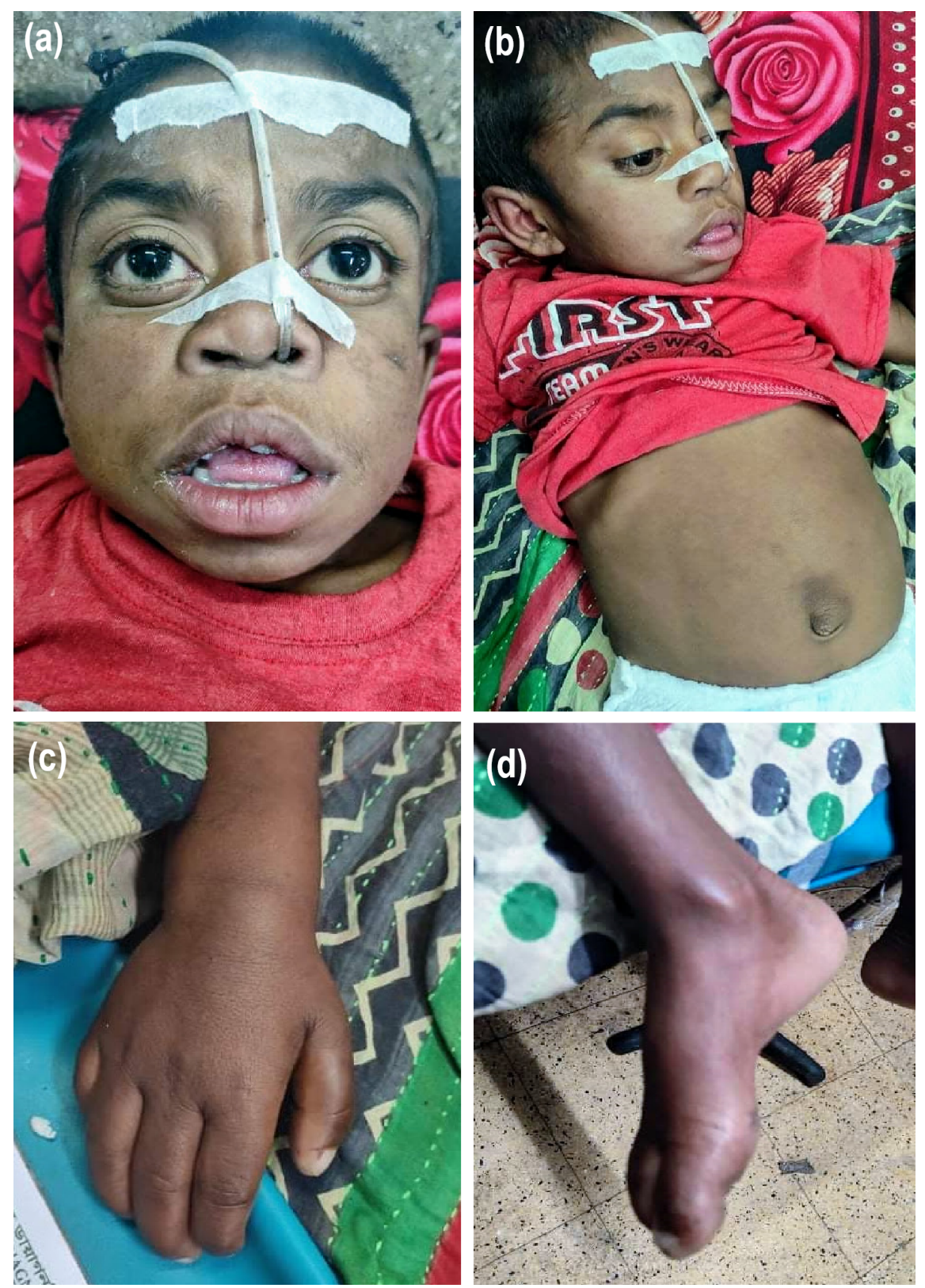


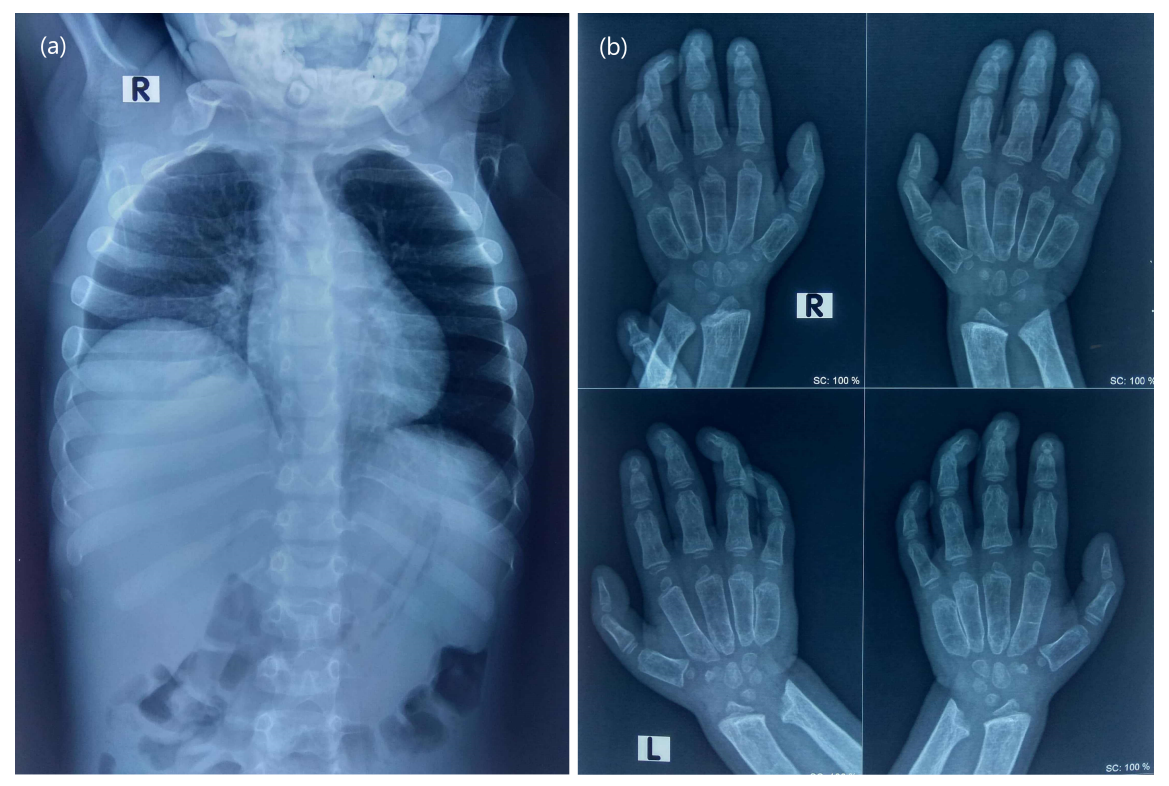

